\title{
Error Modeling and Compensation of Circular Motion on a New Circumferential Drilling System
}

\author{
Qiang Fang, ${ }^{1}$ Yu-Chao Li, ${ }^{1}$ Shao-Hua Fei, ${ }^{1}$ Yong-Wei Han, ${ }^{2}$ and Ying-Lin Ke \\ ${ }^{1}$ State Key Lab of Fluid Power Transmission and Control, School of Mechanical Engineering, Zhejiang University, \\ Hangzhou 310027, China \\ ${ }^{2}$ AVIC Xian Aircraft Industry Group Limited Company, Xian 710089, China
}

Correspondence should be addressed to Qiang Fang; fangqiang@zju.edu.cn

Received 27 March 2015; Revised 28 May 2015; Accepted 30 May 2015

Academic Editor: Qing Chang

Copyright (C) 2015 Qiang Fang et al. This is an open access article distributed under the Creative Commons Attribution License, which permits unrestricted use, distribution, and reproduction in any medium, provided the original work is properly cited.

\begin{abstract}
A new flexible circumferential drilling system is proposed to drill on the fuselage docking area. To analyze the influence of the circular motion error to the drilling accuracy, the nominal forward kinematic model is derived using Denavit-Hartenberg (D-H) method and this model is further developed to model the kinematic errors caused by circular positioning error and synchronization error using homogeneous transformation matrices (HTM). A laser tracker is utilized to measure the circular motion error of the two measurement points at both sides. A circular motion compensation experiment is implemented according to the calculated positioning error and synchronization error. Experimental results show that the positioning error and synchronization error were reduced by $65.0 \%$ and $58.8 \%$, respectively, due to the adopted compensation, and therefore the circular motion accuracy is substantially improved. Finally, position errors of the two measurement points are analyzed to have little influence on the measurement result and the validity of the proposed compensation method is proved.
\end{abstract}

\section{Introduction}

In large aircraft fuselage assembly process, fuselage sections are supported by three-coordination-system numerical positioners $[1,2]$ to align with each other and there are lots of fastener holes to be drilled on the docking area with wide range and high difficulty to join the sections together. Thus automated precision drilling system becomes an essential integral part of aircraft numerical assembly technology. However, traditional approaches for automated drilling of the fuselage sections which employ large truss equipment require high investment and large space during implementation [3]. While this kind of technology remains effective for most applications, the aerospace industry is in demand of developing a low-cost, highly flexible, and lightweight special hole drilling equipment for fuselage sections. The aerospace manufacturing industry has successfully developed such equipment. Boeing Company developed the first Flex Track drilling system; then Electroimpact Company developed a variety of different Flex Track drilling systems to satisfy all kinds of applications, achieving hole accuracy of $0.025 \mathrm{~mm}$. The newly developed Flex Track drilling system in recent years has been successfully applied to the assembly of Boeing's B777 and B787 fuselage sections and Airbus's A380 wing box drilling system [4-6]. The domestic institutes have caught tightly the international steps on the theory research and equipment development of the Flex Track drilling system; among them AVIC Beijing Aeronautical Manufacturing Technology Research Institute developed BAA300 system; this system can ensure the fine accuracy and quality of the holes [7] but is limited to theoretical research and prototype verification; the system has not been put into the practical application. The main advantage of the Flex Track drilling system over traditional drilling system is that Flex Track system uses vacuum cups to install the tracks of the drilling unit directly on the fuselage sections instead of requiring large-scale machine tools like infrastructure. Since the tracks are flexible, they are able to adapt to different machined work pieces, but the precision of the system becomes vulnerable due to the geometry properties of the surface, the structural rigidity, and other factors. Besides, tracks of the Flex Track drilling system need to be installed repeatedly and the geometric error of 
the splice region increases the difficulty of the implementation.

Considering the demand of some fuselage sections splice region hole drilling, based on the features of the existing Flex Track drilling system, Zhejiang University developed a new type of circumferential automated drilling system which has been successfully applied to the production. The circumferential drilling system includes double tracks as the basic system support. The inner circular rail consisting of ten segments is installed on the fuselage surface by adjustable contact pads, and the outer arc guide is used as the support of the drilling actuator. The drilling actuator can move along the outer arc guide and drilling holes within a certain drilling station while the arc guide can move along the circular rails to switch a total of twelve drilling stations. Thus, the circumferential drilling system can complete the whole splice region drilling task through one installation. Closed structure of the circular rails and the double rigid tracks of the system effectively guarantee the system stiffness and constrain fuselage deformation when machined. The adjustable contact pads make the circular rails adapt to large surface curvature, nonround feature, and nonequivalence segment of the drilling surface [7]. The new circumferential automated drilling system introduced in this paper was fully upgraded over the one in literature $[8,9]$ with improved functions and reliability, achieving automatic drilling stations switching and dual-motor synchronous drive for the circular movement axis.

Drilling actuator's position of the circumferential drilling system with respect to the fuselage section is calibrated by the reference holes' position measured by vision-based measurement unit on the arc-based drilling unit. The positions of the holes to be drilled can be planned under the drilling system coordinate system like a $2 \mathrm{D}$ flat problem. While the normality sensor guarantees the normal accuracy of the drilled holes, the position accuracy mainly depends on the accuracy of the circular movement axis and longitudinal movement axis. The circular movement axis differs from traditional revolute axis that it is driven by dual-motor through two gear and arc rack transmission pairs. Due to large motion radius and low rail stiffness, the circular movement axis only engages semiclosed loop control. Therefore, in order to guarantee high ultimate drilling accuracy, it is necessary to improve the circular motion accuracy of the drilling actuator on the arc guide. Electroimpact Company adds a nondrive axis equipped with an encoder to measure the angular difference between the movement along the vacuum track and the longitudinal movement axis in the Flex Track drilling system for B777 fuselage sections assembly in order to eliminate the synchronization error and realize the docking drilling on the round table facet [10-12]. In this solution, the accuracy of effector is determined by the rail on one side and effector's torsional rigidity in the direction of drilling axis is comprised. Thus, it is not suitable for the circumferential drilling system.

Error compensation technology [13-15] is applied to CNC machine tools first and can be utilized to improve the circular motion accuracy of the circumferential drilling system. Three main error sources $[16,17]$ of CNC machine tools are geometric and kinematic errors, thermal errors, and cutting force induced errors, among which geometric and kinematic errors typically account for more than $40 \%$ with good repeatability. The circumferential drilling system has a lower movement velocity compared to CNC machine tools, and other movement axes remain steady while drilling holes so that they produce lower thermal errors and cutting forces errors. Therefore, compensation for geometric errors can effectively improve the accuracy of movement of the drilling actuator. Error compensation technique includes error modeling, error measurement, and error compensation. As the theoretical foundation of error measurement and compensation, establishing accurate comprehensive error model can effectively evaluate the influence of each error component, the normal ways of modeling are geometric modeling, Homogeneous coordinate transformation matrix, and modeling based on multibody system theory. The measurement system with laser tracker is of the advantages of fast and high accuracy, and common equipment for error detection fits to this system to measure the points in a large space. Considering the error after measuring and identifying, the opposite offset has been added into the software in advance, in order to do the compensation of the system.

In Section 2 the structure of the proposed circumferential drilling system is analyzed. The nominal forward kinematic model is derived and this model is further developed to model the kinematic errors caused by circular positioning error and synchronization error using the homogeneous coordinate transformation method in Section 3. The compensation method is introduced according to Section 4 . To verify the compensation method, the compensation experiment has been performed with the utilization of a laser tracker and the experiment result is discussed in Section 5.

\section{Structure of the Circumferential Drilling System}

The circumferential drilling system is newly proposed, highly flexible, and lightweight numerical drilling equipment for the demand of drilling on the fuselage sections splice region based on a comprehensive analysis of the traditional drilling machine and contemporary Flex Track system. The main structure of the circumferential drilling system consists of bottom auxiliary carriage, circular rails, holders, and arc-base drilling unit, equipped with vacuum cleaner, cooling system, and controller cart, as shown in Figure 1.

The bottom auxiliary carriage is the main support for circular rails. The circular rails are installed on the fuselage sections by contact pads, and the arc-base drilling unit is mounted on the circular rails.

Arc-base drilling unit is used for drilling in the splice region within an arc's span, containing six axes of motion, as shown in Figure 2. The $X$-axis along the arc guide is driven by the dual-motor with the constraint of the arc guide. The arc guide occupies range of $53^{\circ}$ to ensure finishing the drilling task of the entire circumferential region in twelve drilling stations and keeping space for station switching. The $Y$-axis moves along the longitudinal direction. The $X$-axis and $Y$ axis are used to control the position of the holes. The $A$-axis 


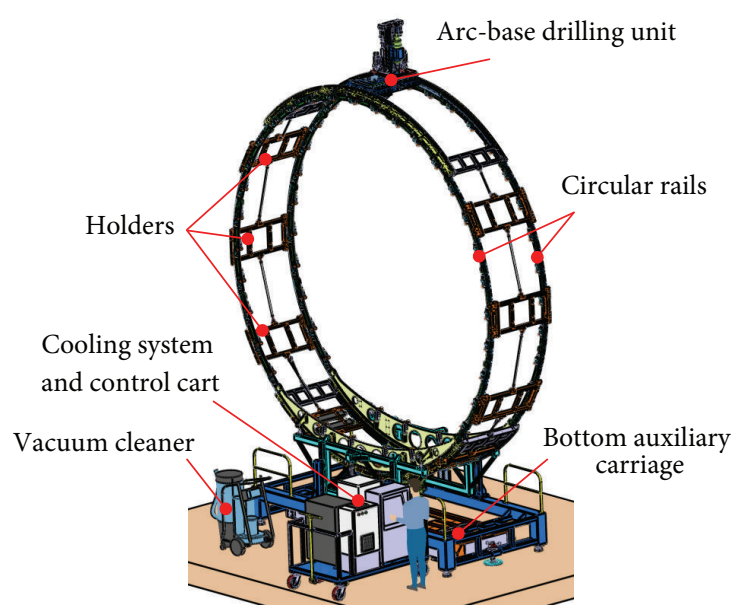

FIGURE 1: Structure of circumferential drilling machine.

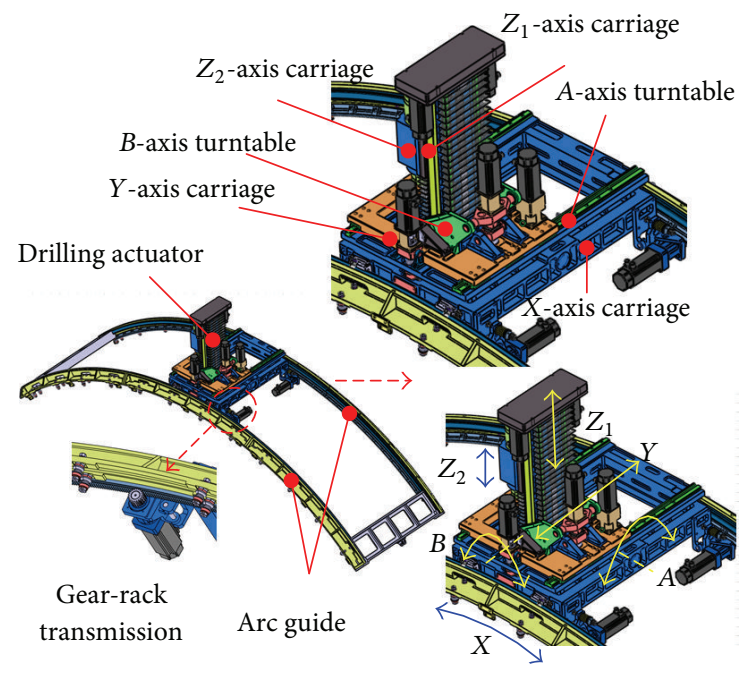

FIgURe 2: Arc-base drilling unit.

offers rotation about the tangent line of the $X$-axis and the $B$-axis offers rotation about the $Y$-axis. The $A$-axis and $B$ axis both control the normality of the drilled holes. The two $Z$-axes move along the drilling direction. The $Z_{1}$-axis with larger motion range is used to avoid obstacles and make space for tool exchange, while the feeding $Z_{2}$-axis can guarantee precise countersink depth by controlling the relative position with the pressure foot. The drilling unit is mounted with the normality sensor and the vision-based measurement unit.

Since the drilling actuator can only drill holes within an arc's span of $30^{\circ}$, the arc-base drilling unit needs to be fixed on the circular rails at different angle to finish the drilling task. The automated drilling station switching process is shown in Figure 3. When the drilling unit drills holes within current station, the arc guide is locked on the circular rails by one group of electromagnetic latches. When the arc-base drilling unit needs to switch to the next station, the drilling actuator moves to specified position and the other group of electromagnetic latches locks the drilling actuator on the circular rails; with the first group of electromagnetic latches

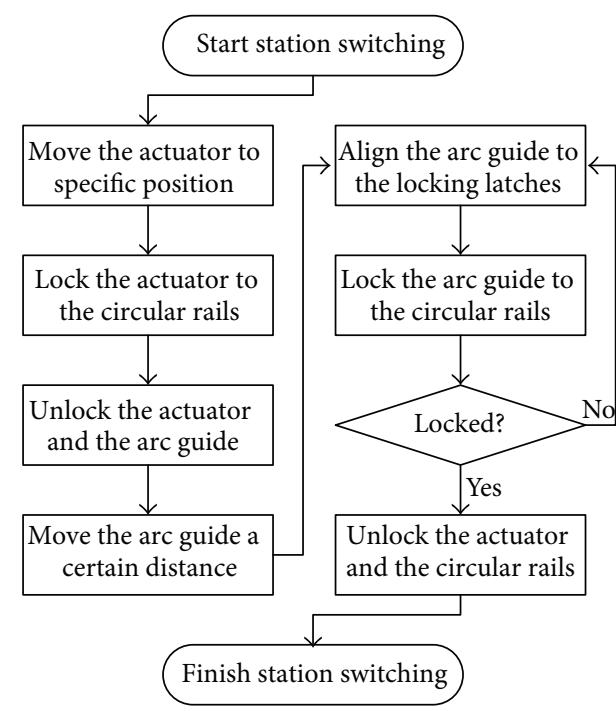

FIGURE 3: Drilling station switch process.

released, the arc guide moves along the circular rails driven by the dual-motor to switch drilling station. Therefore, the circumferential drilling system can finish drilling task of the entire splice region automatically.

\section{Circular Motion Error Modeling}

Error modeling is not only an effective way to analyze the causes of error but also the theoretical basis for improving the drilling accuracy through error compensation. The arc-base drilling unit contains six movement axes: $X$-axis, $A$-axis, $Y$ axis, $B$-axis, $Z_{1}$-axis, and $Z_{2}$-axis according to the order of the kinematic chain. Denavit-Hartenberg (D-H) method is adopted to derive the nominal forward kinematic model of the drilling machine [8], as shown in Figure 4.

There are six joint axes in the kinematic model of the circumferential drilling system, denoted as axis $i, i=$ $1,2, \ldots, 6$, where axis $i$ describes motion between link $i-1$ and link $i$ and frame $\{i\}$ is attached rigidly to link $i$. Under $\mathrm{D}$-H notation, the $\widehat{Z}$-axis of frame $\{i\}$ called $\widehat{Z}_{i}$ is coincident with the motion direction of axis $i$ and $\widehat{X}_{i}$ points along $a_{i}$ from axis $i$ to axis $i+1$, where $a_{i}$ is the length of the common perpendicular between axis $i$ and axis $i+1$.

For convenience, base frame $\{0\}$ attached to the base of the circumferential drilling system is chosen coincident to frame $\{1\}$ when axis 1 is in zero position. The origin of the frame $\{0\}$ is located right between the centers of the two circular rails. Axis 1 is the revolute $X$-axis and frame $\{1\}$ is rotated from frame $\{0\}$ by the joint variable $\theta_{1}$. The pose of frame $\{1\}$ with respect to frame $\{0\}$ is

$$
{ }_{1}^{0} T=R_{z}\left(\theta_{1}\right)=\left[\begin{array}{cccc}
c \theta_{1} & -s \theta_{1} & 0 & 0 \\
s \theta_{1} & c \theta_{1} & 0 & 0 \\
0 & 0 & 1 & 0 \\
0 & 0 & 0 & 1
\end{array}\right],
$$

where $s(\cdot)$ and $c(\cdot)$ denote $\sin (\cdot)$ and $\cos (\cdot)$, respectively. 

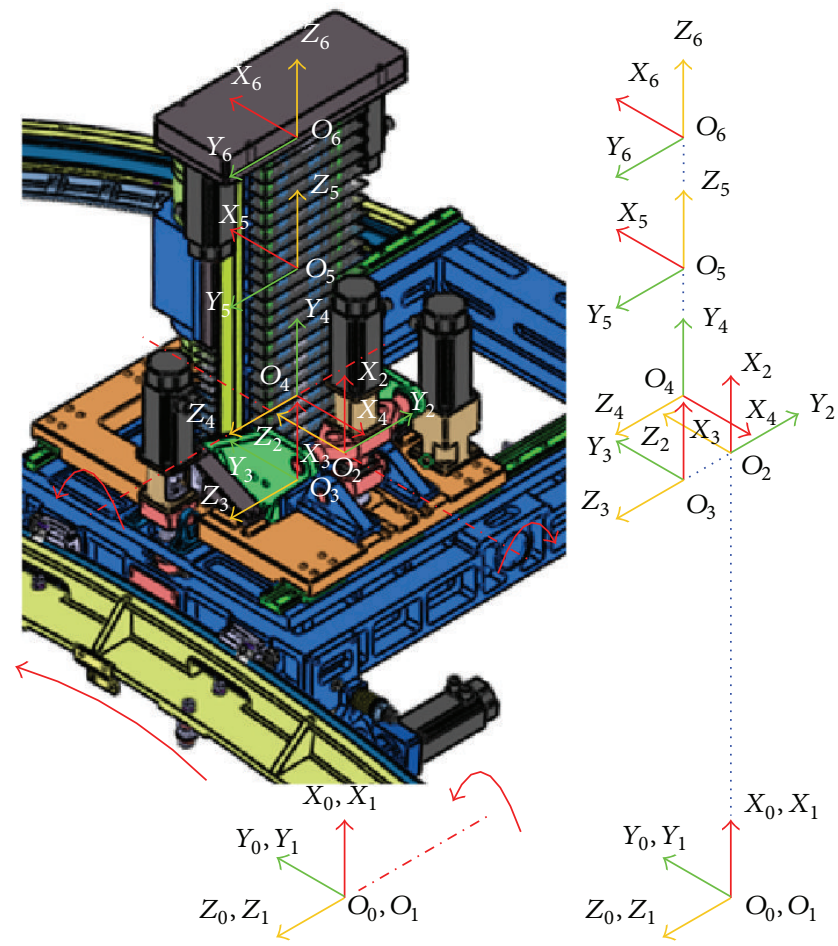

FIGURE 4: Kinematic structure of drilling unit.

Axis 2 is the rotational $A$-axis about the tangent line of the $X$-axis. Frame $\{2\}$ can be acquired in three steps:

(1) Translate frame $\{1\}$ along $\widehat{X}_{1}$ by $a_{1}$ (radius of the $X$ axis).

(2) Rotate around $\widehat{X}_{1}$ by $-90^{\circ}$.

(3) Rotate around $\widehat{Z}_{1}$ by joint variable $\theta_{2}$.

The pose of frame $\{2\}$ with respect to frame $\{1\}$ is

$$
\begin{aligned}
{ }_{2}^{1} T & =T_{x}\left(a_{1}\right) R_{x}\left(-90^{\circ}\right) R_{z}\left(\theta_{2}\right) \\
& =\left[\begin{array}{cccc}
c \theta_{2} & -s \theta_{2} & 0 & a_{1} \\
0 & 0 & 1 & 0 \\
-s \theta_{2} & -c \theta_{2} & 0 & 0 \\
0 & 0 & 0 & 1
\end{array}\right] .
\end{aligned}
$$

Axis 3 is the translational $Y$-axis along the longitudinal direction. Frame $\{3\}$ can be acquired in two steps:

(1) Rotate frame $\{2\}$ around $\widehat{X}_{2}$ by $90^{\circ}$.

(2) Translate along $\widehat{Z}_{2}$ by joint variable $d_{3}$.

The pose of frame $\{3\}$ with respect to frame $\{2\}$ is

$$
{ }_{3}^{2} T=R_{x}\left(90^{\circ}\right) T_{z}\left(d_{3}\right)=\left[\begin{array}{cccc}
1 & 0 & 0 & 0 \\
0 & 0 & -1 & -d_{3} \\
0 & 1 & 0 & 0 \\
0 & 0 & 0 & 1
\end{array}\right] .
$$

Axis 4 is the rotational $B$-axis about the $Y$-axis. Frame $\{4\}$ can be acquired in three steps:

(1) Translate frame $\{3\}$ along $\widehat{X}_{3}$ by $a_{3}$ (distance between $A$-axis and $B$-axis)

(2) Rotate around $\widehat{Z}_{3}$ by $-90^{\circ}$.

(3) Rotate around $\widehat{Z}_{3}$ by joint variable $\theta_{4}$.

The pose of frame $\{4\}$ with respect to frame $\{3\}$ is

$$
{ }_{4}^{3} T=T_{x}\left(a_{3}\right) R_{z}\left(-90^{\circ}\right) R_{z}\left(\theta_{4}\right)\left[\begin{array}{cccc}
s \theta_{4} & c \theta_{4} & 0 & a_{3} \\
-c \theta_{4} & -s \theta_{4} & 0 & 0 \\
0 & 0 & 1 & 0 \\
0 & 0 & 0 & 1
\end{array}\right] \text {. }
$$

Axis 5 and axis 6 are the $Z_{1}$-axis and the $Z_{2}$-axis moving along the drilling direction, respectively. Frame $\{5\}$ can be acquired in three steps:

(1) Rotate frame $\{4\}$ around $\widehat{Z}_{4}$ by $180^{\circ}$.

(2) Rotate around $\widehat{X}_{4}$ by $90^{\circ}$.

(3) Translate along $\widehat{Z}_{4}$ by joint variable $d_{5}$.

The pose of frame $\{5\}$ with respect to frame $\{4\}$ is

$$
{ }_{5}^{4} T=R_{z}\left(180^{\circ}\right) R_{x}\left(90^{\circ}\right) T_{z}\left(d_{5}\right)=\left[\begin{array}{cccc}
-1 & 0 & 0 & 0 \\
0 & 0 & 1 & d_{5} \\
0 & 1 & 0 & 0 \\
0 & 0 & 0 & 1
\end{array}\right] \text {. }
$$

And frame $\{6\}$ is translated from frame $\{5\}$ along $\widehat{Z}_{5}$ by joint variable $d_{6}$. The pose of frame $\{6\}$ with respect to frame $\{5\}$ is

$$
{ }_{6}^{5} T=T_{z}\left(d_{6}\right)=\left[\begin{array}{cccc}
1 & 0 & 0 & 0 \\
0 & 1 & 0 & 0 \\
0 & 0 & 1 & d_{6} \\
0 & 0 & 0 & 1
\end{array}\right]
$$

In order to determine the coordinate of the tool center point with respect to the base frame $\{0\}$, frame $\{7\}$ is attached to the tool with fixed position offset $\left(x_{7}, y_{7}, z_{7}\right)$ with respect to frame $\{6\}$. The pose of frame $\{7\}$ with respect to frame $\{6\}$ is

$$
{ }_{7}^{6} T=\left[\begin{array}{cccc}
1 & 0 & 0 & x_{7} \\
0 & 1 & 0 & y_{7} \\
0 & 0 & 1 & z_{7} \\
0 & 0 & 0 & 1
\end{array}\right]
$$

Link parameters of the arc-base drilling unit are shown in Table 1 . 
The nominal forward kinematic model of the circumferential drilling system can be derived by multiplying coordinate transformation matrix (1) to (7) as follows:

$$
{ }_{7}^{0} T=\prod_{i=1}^{7}\left({ }^{i-1} T\right) .
$$

When the drilling actuator moves along the arc guide, there are six geometric error components in all. Since circular positioning error and synchronization error can only be compensated by the dual-motor, the other errors are small and can only be reduced by other axes. Hence, the circular dual-drive positioning error and synchronization error are considered to establish comprehensive error model to do compensation on the dual-motor. The parameters of the circular movement axis are shown in Table 2, where $R$ is the radius of the arc rack, $L$ is the distance between the two racks, and $D$ is the travel distance of the arc guide.

Note $x_{1}$ and $x_{2}$ as the actual displacements of the drilling actuator on the two racks, respectively. The circular positioning error causes angle error $\Delta \gamma$ around $\widehat{Z}_{1}$ of frame $\{1\}$ :

$$
\Delta \gamma=\frac{\left(\left(x_{1}+x_{2}\right) / 2-x_{\mathrm{cmd}}\right)}{R} .
$$

The circular synchronization error causes angle error $\Delta \alpha$ around $\widehat{X}_{1}$ of frame $\{1\}$ :

$$
\Delta \alpha=\arctan \left(\frac{x_{1}-x_{2}}{L}\right)
$$

Therefore, after taking circular positioning error and synchronization error into consideration, transform ${ }_{1}^{0} \mathrm{~T}$ is replaced by ${ }_{1}^{0} T^{\prime}$ :

$$
{ }_{1}^{0} T^{\prime}={ }_{1}^{0} T \cdot R_{\widehat{Z}_{1}}(\Delta \gamma) \cdot R_{\widehat{X}_{1}}(\Delta \alpha),
$$

where

$$
\begin{aligned}
R_{\widehat{Z}_{1}}(\Delta \gamma)= & {\left[\begin{array}{cccc}
c \Delta \gamma & -s \Delta \gamma & 0 & 0 \\
s \Delta \gamma & c \Delta \gamma & 0 & 0 \\
0 & 0 & 1 & 0 \\
0 & 0 & 0 & 1
\end{array}\right] } \\
R_{\widehat{X}_{1}}(\Delta \alpha) & =\left[\begin{array}{cccc}
1 & 0 & 0 & 0 \\
0 & c \Delta \alpha & -s \Delta \alpha & 0 \\
0 & s \Delta \alpha & c \Delta \alpha & 0 \\
0 & 0 & 0 & 1
\end{array}\right] .
\end{aligned}
$$

Thus, the forward kinematic model of the circumferential drilling system considering circular motion error is

$$
{ }_{7}^{0} T^{\prime}={ }_{1}^{0} T^{\prime} \prod_{i=2}^{7}\left({ }_{i}^{i-1} T\right) .
$$

TABLE 1: Link parameters of the arc-base drilling unit.

\begin{tabular}{lccccc}
\hline Link $i$ & $a_{i-1}$ & $\alpha_{i-1}$ & $d_{i}$ & $\theta_{i}$ & Joint variable \\
\hline 1 & 0 & $0^{\circ}$ & 0 & $\theta_{1}$ & $\theta_{1}: \pm 16^{\circ}$ \\
2 & $a_{1}(2985 \mathrm{~mm})$ & $-90^{\circ}$ & 0 & $\theta_{2}$ & $\theta_{2}: \pm 10^{\circ}$ \\
3 & 0 & $90^{\circ}$ & $d_{3}$ & $0^{\circ}$ & $d_{3}: 450 \mathrm{~mm}$ \\
4 & $a_{3}(170 \mathrm{~mm})$ & $180^{\circ}$ & 0 & $\theta_{3}$ & $\theta_{3}: \pm 15^{\circ}$ \\
5 & 0 & $90^{\circ}$ & $d_{5}$ & $180^{\circ}$ & $d_{5}: 500 \mathrm{~mm}$ \\
6 & 0 & $180^{\circ}$ & $d_{6}$ & $0^{\circ}$ & $d_{6}: 300 \mathrm{~mm}$ \\
\hline
\end{tabular}

And the tool center positioning error is

$$
E={ }_{7}^{0} T^{\prime}-{ }_{7}^{0} T .
$$

Therefore, according to the circumferential drilling system forward kinematic model and parameters, given circular positioning error and synchronization error, tool center positioning error can be calculated to evaluate the influence to the ultimate drilling accuracy.

\section{Error Compensation Methodology}

Error compensation refers to the method of artificially creating a new error to neutralize the systematic error of the current movement. The circular movement axis of the circumferential drilling system generates circular paths. Due to small central angle of the path, arc fitting will introduce large errors. Hence, chord lengths of the adjacent points are used to calculate positioning error and synchronization error. Also, the average chord length $c_{\mathrm{avg}}$ is used as the ideal chord length:

$$
c_{\text {avg }}=\frac{\left(\sum_{i=1}^{N} c_{1 i}+\sum_{i=1}^{N} c_{2 i}\right)}{2 N},
$$

where $c_{1 i}$ and $c_{2 i}$ are the chord lengths of the adjacent points on the two rails, respectively, and $N$ is the quantity of chords of each rail.

Circular positioning error $\Delta_{n}$ is calculated by

$$
\Delta_{n}=\sum_{i=1}^{n} \frac{c_{1 i}+c_{2 i}}{2}-c_{\mathrm{avg}} \cdot n,
$$

where $n=1,2, \ldots, N$ is the number of measurement points.

Circular synchronization error $\xi_{n}$ is calculated by

$$
\xi_{n}=\left.\left(c_{1 i}-c_{2 i}\right)\right|_{i=n} .
$$

Compensation value is calculated as follows:

$$
\begin{aligned}
& x_{\mathrm{cmd} 1 n}^{\prime}=x_{\mathrm{cmd} n} \cdot \frac{c_{\mathrm{avg}} \cdot n}{\sum_{i=1}^{n} c_{1 i}} \\
& x_{\mathrm{cmd} 2 n}^{\prime}=x_{\mathrm{cmd} n} \cdot \frac{c_{\mathrm{avg}} \cdot n}{\sum_{i=1}^{n} c_{2 i}},
\end{aligned}
$$

where $x_{\mathrm{cmd} 1 n}^{\prime}$ and $x_{\mathrm{cmd} 2 n}^{\prime}$ are the compensation value of the two rails, respectively, and $x_{\mathrm{cmd} n}$ is the demand value before compensation. The compensation value between measurement points is calculated by linear interpolation. 
TABLE 2: Parameters of circular movement axis.

\begin{tabular}{lcccccc}
\hline Name & $R$ & $L$ & $D$ & Reduction ratio & Motor type & Gear parameters \\
\hline Value & $2900 \mathrm{~mm}$ & $942 \mathrm{~mm}$ & $1600 \mathrm{~mm}$ & 25 & AKM32E-ANC2C-00 & $d=40 \mathrm{~mm}, m=2$ \\
\hline
\end{tabular}

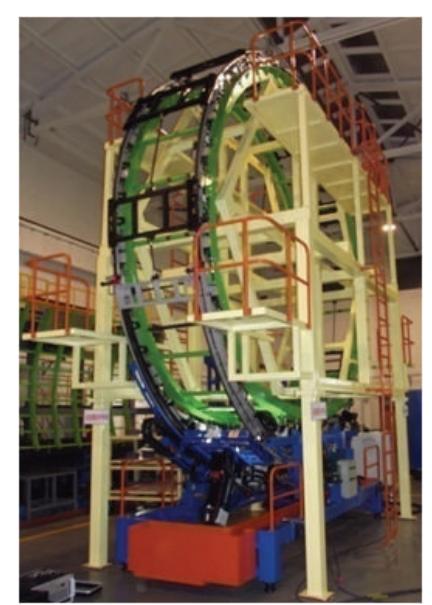

FIgURE 5: The circumferential drilling machine.

\section{Experiment and Discussion}

In order to verify the compensation method, a compensation experiment is perforated on the circumferential drilling system as shown in Figure 5. A Leica laser tracker is adopted to measure the circular positioning error and synchronization error when the drilling actuator is moving along the arc guide, as shown in Figure 6.

Here is the experiment procedure:

(1) Select a proper drilling station and pick two measurement points symmetrically on both sides of the drilling actuator near the circular rails.

(2) Within the whole $1600 \mathrm{~mm}$ range of the $X$-axis, measure both sides of the measurement points every $50 \mathrm{~mm}$ movement.

(3) Repeat (2) three times and take an average to gain 32 pairs of point coordinates approximate on two parallel arc segments.

(4) Use 31 pairs of chord lengths of the adjacent points to calculate positioning error and synchronization error for compensation value calculation.

(5) Repeat (3) and (4) using compensation value and compare the positioning error and synchronization error with the ones before compensation.

Error compensation implementation intends to set the tool or work piece position to generate relative motion in the reverse error direction to eliminate the original error. Software compensation technology will set offset on the original command value in advance which is economic and convenient to implement. The circumferential drilling system movement axes use Kollmorgen motion solutions and the drilling control system is developed with the provided MPI
Circumferential drilling system

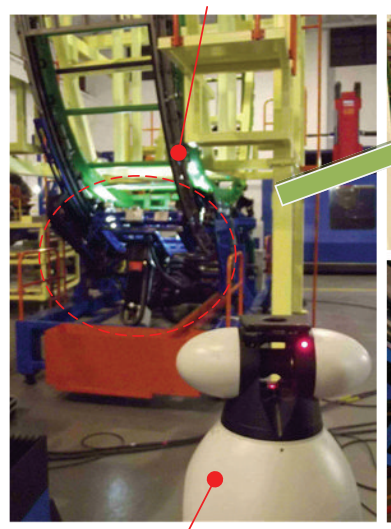

Leica laser tracker
Arc-base drilling unit

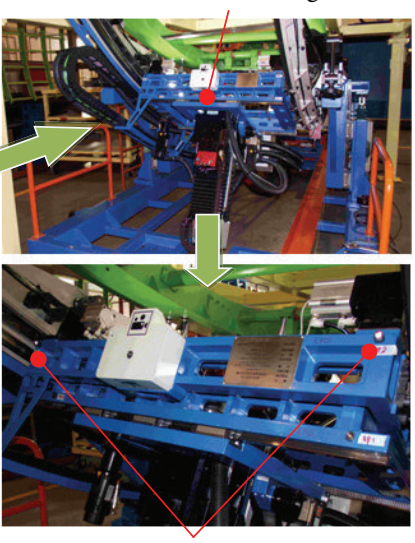

Measurement points
FIgURE 6: Compensation experiment.

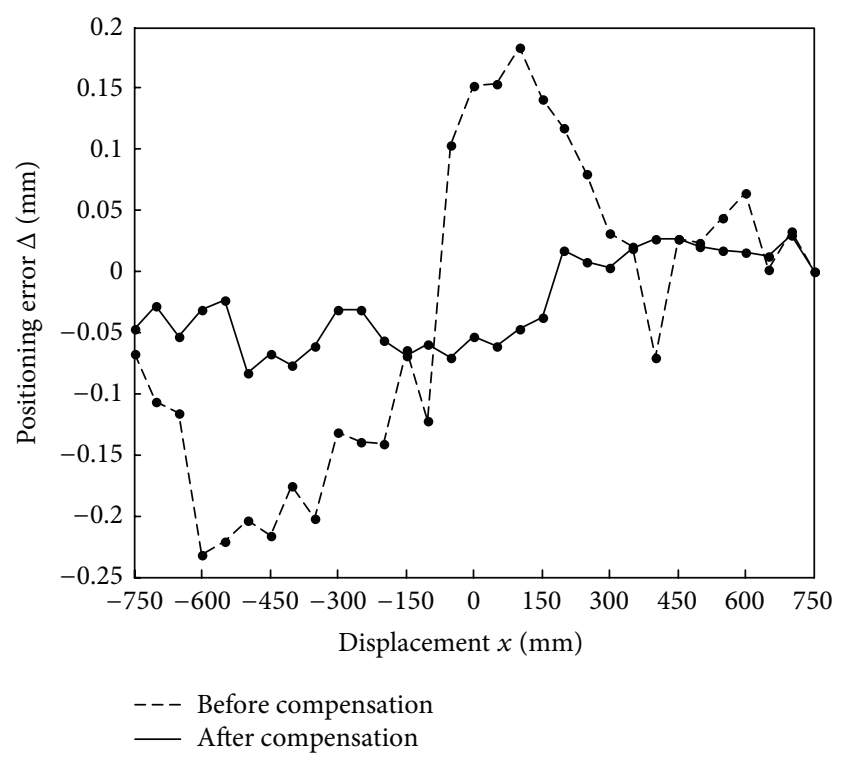

FIGURE 7: Positioning error comparison.

(motion programming interface) in $\mathrm{C}++$ /MFC environment. Hence, compensation program can be easily developed.

Do compensation experiment with compensation value calculated according to formula (19); the comparison of the positioning error and synchronization error before and after compensation is shown in Figures 7 and 8, respectively. The circular positioning error and synchronization error were reduced by $65.0 \%$ and $58.8 \%$, respectively, after compensation. Thus, the circular motion accuracy is largely improved due to the compensation.

In general, only one measurement point is utilized when using a laser tracker to measure position. In order to measure 


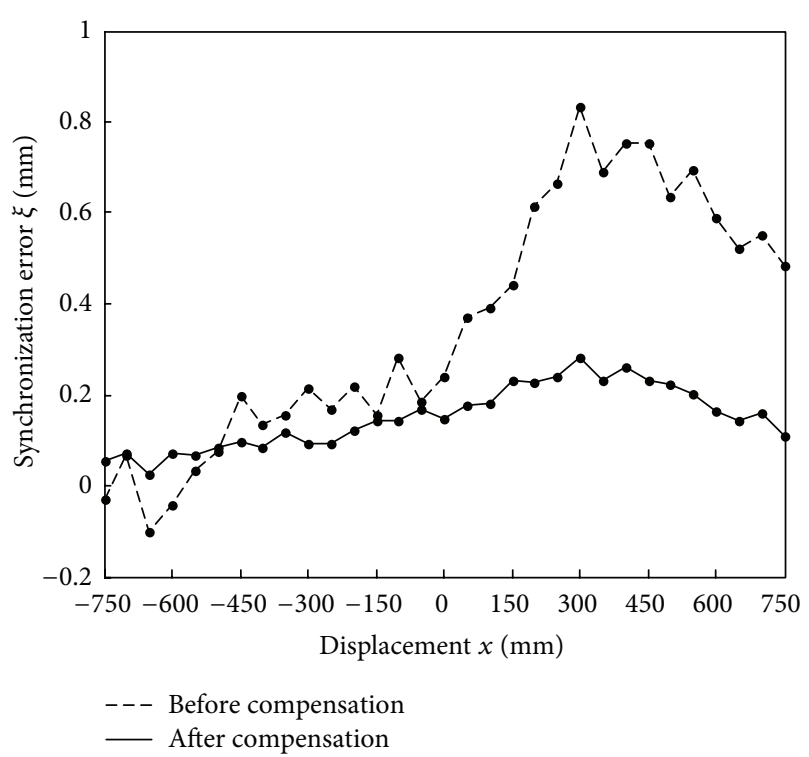

FIGURE 8: Synchronous error comparison.

synchronization error, two measurement points are adopted here. It is essential to analyze the influence of the position errors of the two measurement points, as shown in Figure 9.

Without loss of generality, assume that one of the measurement points is in ideal position, the other measurement point has spatial position error $\delta=\left(\delta_{x}, \delta_{y}, \delta_{z}\right)$ in frame $\{1\}$, where $\delta_{x}$ and $\delta_{y}$ cause deviation along the radius direction and generate additional synchronization error. $\delta_{z}$ along the longitude direction changes distance to the plane $X_{1} O_{1} Y_{1}$ and magnify synchronization error by $\left(L_{1}-\delta_{z}\right) / L_{1}$, where $L_{1}$ is the nominal distance between two measurement points. In summary, the measured synchronization error $\xi_{n}^{\prime}$ and the actual synchronization error $\xi_{n}$ have the following relationship:

$$
\begin{aligned}
\xi_{n}^{\prime}= & \sum_{i=1}^{n} \frac{\left(c_{1 i}+c_{2 i}\right)\left(\delta_{x} \cos \alpha-\delta_{y} \sin \alpha\right)}{2 R_{1}} \\
& +\frac{\left(L_{1}-\delta_{z}\right) \cdot \xi_{n}}{L_{1}}
\end{aligned}
$$

where $R_{1}$ is the nominal motion radius of the measurement points and $\alpha$ is the angle of the measurement points in plane $X_{1} O_{1} Y_{1}$. Since $\alpha$ is very small and $L_{1} \gg \delta_{z}$, formula (20) can be simplified to

$$
\xi_{n}^{\prime}-\xi_{n}=\sum_{i=1}^{n} \frac{\left(c_{1 i}+c_{2 i}\right) \delta_{x}}{2 R_{1}}
$$

Suppose $\delta_{x}=0.2 \mathrm{~mm}$; according to formula (21), measurement error induced synchronization error is less than $0.1 \mathrm{~mm}$. Therefore, $\delta$ has little influence on the measurement result so that the measurement and compensation can effectively improve the circular motion accuracy within a certain precision.

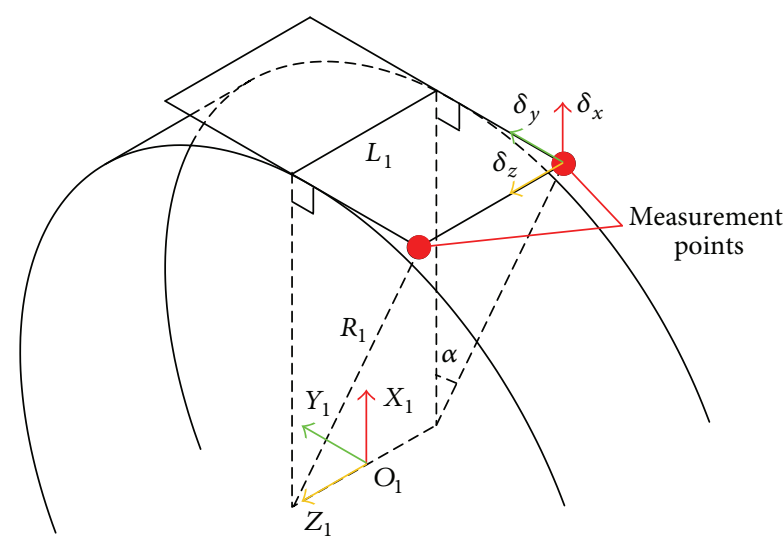

FIGURE 9: Measurement error analysis.

\section{Conclusion}

This paper describes a newly developed circumferential drilling system to satisfy fuselage section splice region drilling demand and derives the nominal forward kinematic model. Comprehensive error model is established by using the homogeneous coordinate transformation considering circular positioning error and synchronization error. The laser tracker is utilized to measure the circular motion error and the compensation experiment is implemented. The experimental result shows that the positioning error and synchronization error were reduced by $65.0 \%$ and $58.8 \%$, respectively. At last, position errors of the two measurement points are analyzed to have little influence on the measurement result and the proposed compensation method is effective.

\section{Conflict of Interests}

The authors declare that there is no conflict of interests regarding the publication of this paper.

\section{References}

[1] B.-G. Qiu, J.-X. Jiang, and Y.-L. Ke, "A new principle and device for large aircraft components gaining accurate support by ball joint," Journal of Zhejiang University: Science A, vol. 12, no. 5, pp. 405-414, 2011.

[2] B. Zhang, B.-G. Yao, and Y.-L. Ke, "A novel posture alignment system for aircraft wing assembly," Journal of Zhejiang University: Science A, vol. 10, no. 11, pp. 1624-1630, 2009.

[3] P. Thompson, J. Hartmann, E. Feikert, and J. Buttrick, "Flex track for use in production," SAE Technical Paper, 2005.

[4] R. S. Wright, C. J. Erickson, and J. J. Jimenez, "United States Patent: 7794183-Burrless flexible track drilling system and method having counterweight tool balancing system," 7794183, 2010.

[5] S. Hogan, J. Hartmann, B. Thayer et al., "Automated wing drilling system for the A380-GRAWDE," SAE Technical Paper 2003-01-2940, SAE International, Warrendale, Pa, USA, 2003.

[6] P. Thompson, H. Oberoi, and A. Draper, "Development of a multi spindle flexible drilling system for circumferential splice 
drilling applications on the 777 airplane," SAE Technical Paper, 2008.

[7] W. Qu, L. Fang, Y. Ke, W. Fang, S. Liu, and H. Xing, "Analysis of locating scheme for the circumferential flex track drilling system," Acta Aeronautica Et Astronautica Sinica, vol. 35, no. 8, pp. 2319-2330, 2014.

[8] W. Zhu, B. Mei, and Y. Ke, "Kinematic modeling and parameter identification of a new circumferential drilling machine for aircraft assembly," The International Journal of Advanced Manufacturing Technology, vol. 72, no. 5-8, pp. 1143-1158, 2014.

[9] W. Zhu, B. Mei, and Y. Ke, "Inverse kinematics solution of a new circumferential drilling machine for aircraft assembly," Robotica, 2014.

[10] H. Oberoi, A. Draper, and P. Thompson, "Production implementation of a multi spindle flexible drilling system for circumferential splice drilling applications on the 777 airplane," SAE Technical Paper 2009-01-3090, 2009.

[11] B. Seater, 5-Axis Flex Track System, 2012-01-1859, SAE International, Warrendale, Pa, USA, 2012.

[12] J. R. Malcomb, "5-axis flex track drilling systems on complex contours: solutions for position control," SAE Technical Paper 2013-01-2224, SAE International, Warrendale, Pa, USA, 2013.

[13] K. F. Eman, B. T. Wu, and M. F. DeVries, "A generalized geometric error model for multi-axis machines," CIRP AnnalsManufacturing Technology, vol. 36, no. 1, pp. 253-256, 1987.

[14] R. Ramesh, M. A. Mannan, and A. N. Poo, "Error compensation in machine tools-a review. Part I: geometric, cutting-force induced and fixture-dependent errors," International Journal of Machine Tools and Manufacture, vol. 40, no. 9, pp. 1235-1256, 2000.

[15] S. Sartori and G. X. Zhang, "Geometric error measurement and compensation of machines," CIRP Annals-Manufacturing Technology, vol. 44, no. 2, pp. 599-609, 1995.

[16] N. A. Barakat, M. A. Elbestawi, and A. D. Spence, "Kinematic and geometric error compensation of a coordinate measuring machine," International Journal of Machine Tools and Manufacture, vol. 40, no. 6, pp. 833-850, 2000.

[17] S. Aguado, D. Samper, J. Santolaria, and J. J. Aguilar, "Identification strategy of error parameter in volumetric error compensation of machine tool based on laser tracker measurements," International Journal of Machine Tools and Manufacture, vol. 53, no. 1, pp. 160-169, 2012. 


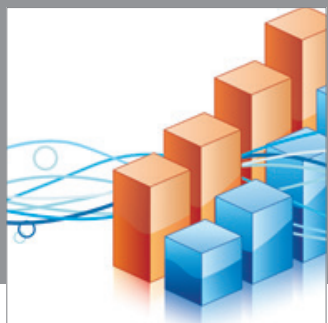

Advances in

Operations Research

mansans

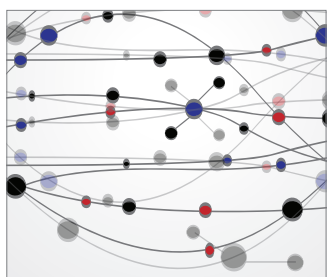

The Scientific World Journal
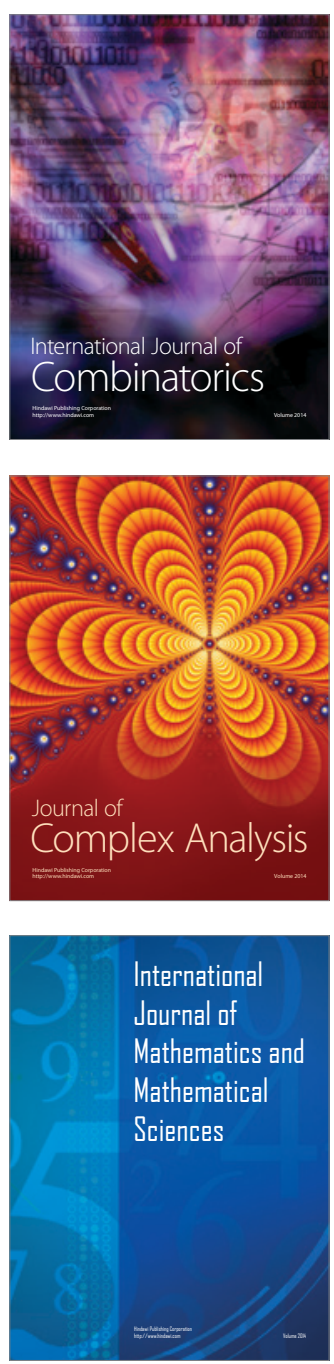
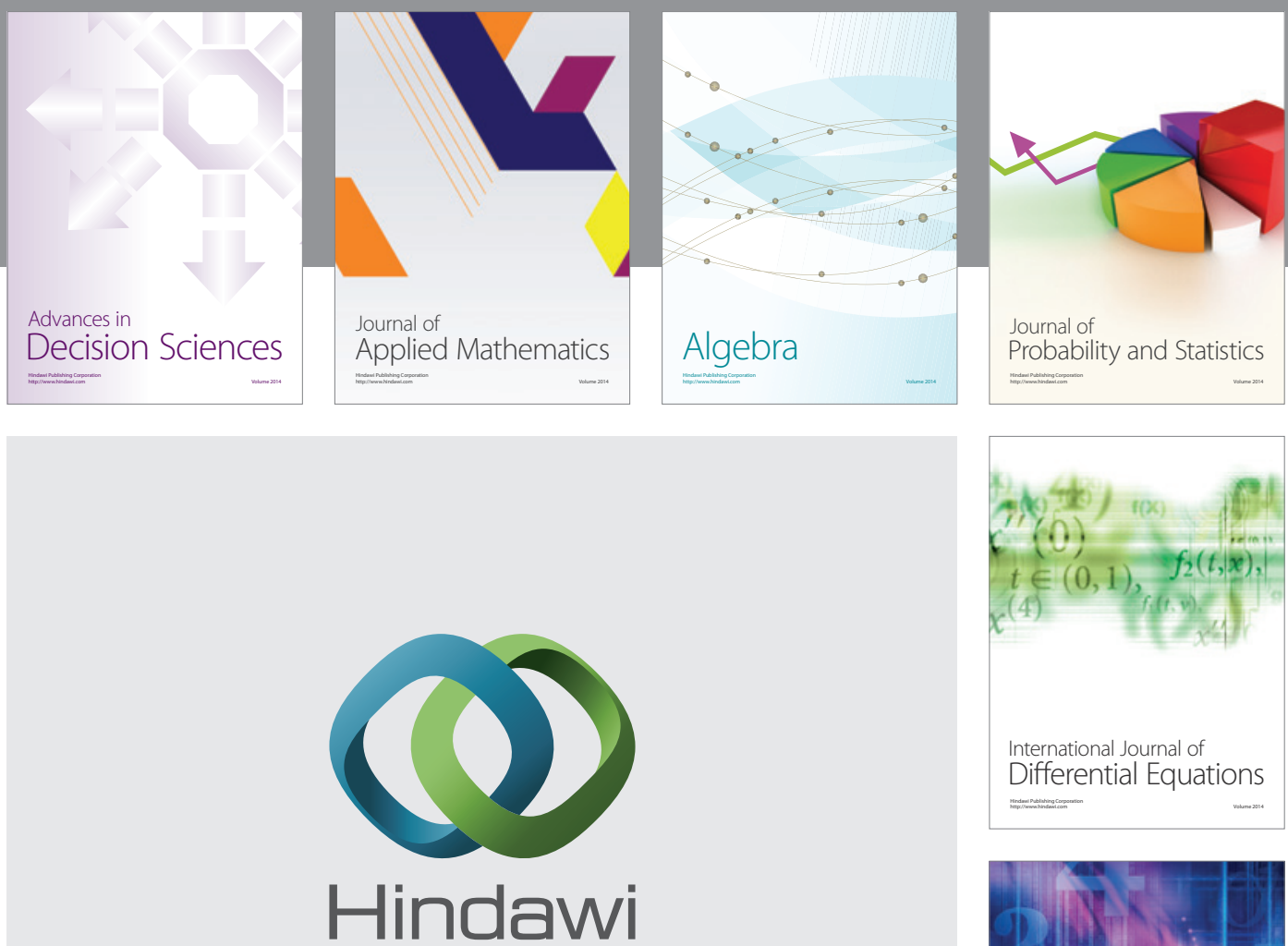

Submit your manuscripts at http://www.hindawi.com
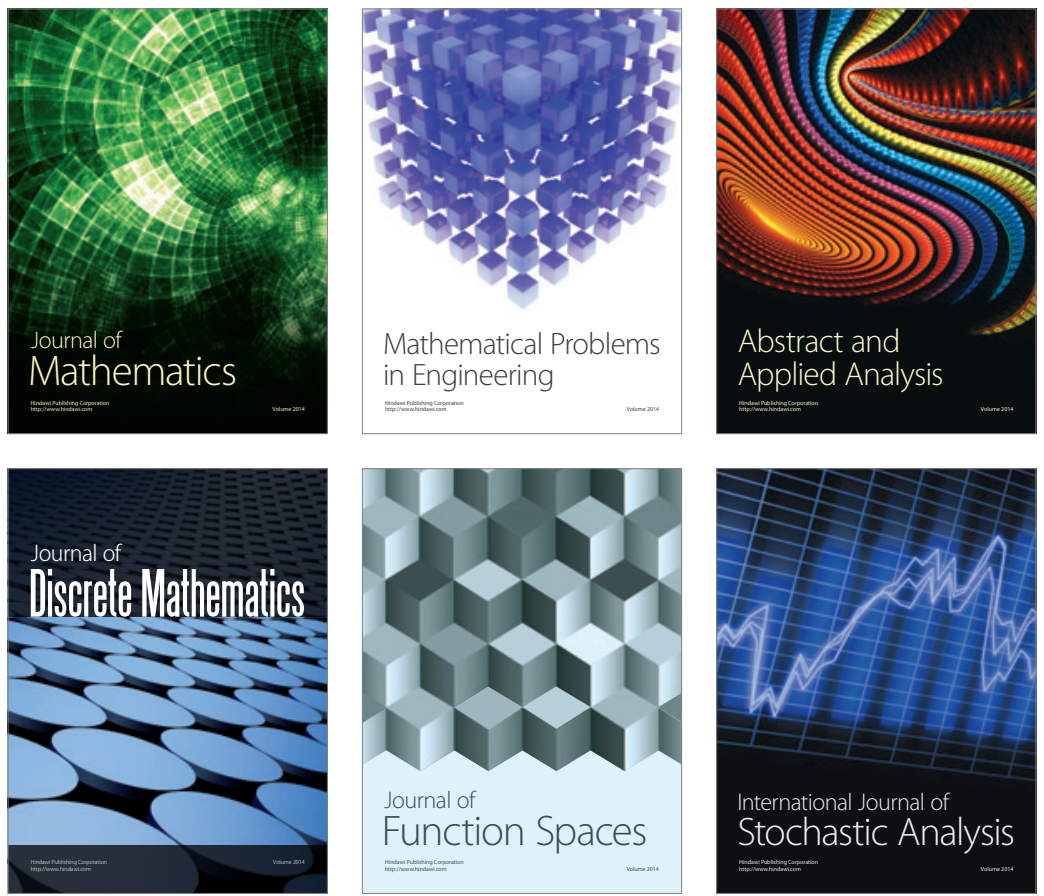

Journal of

Function Spaces

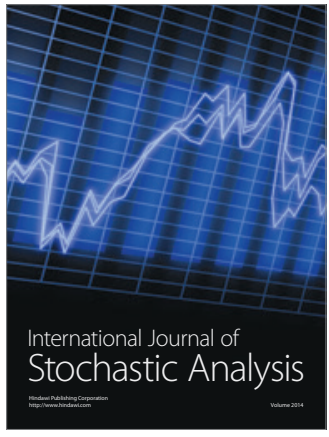

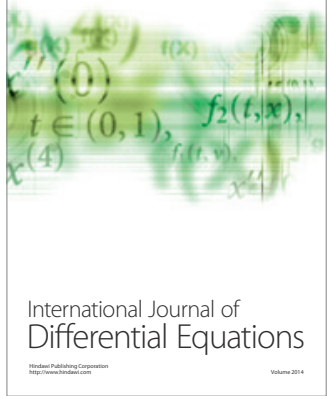
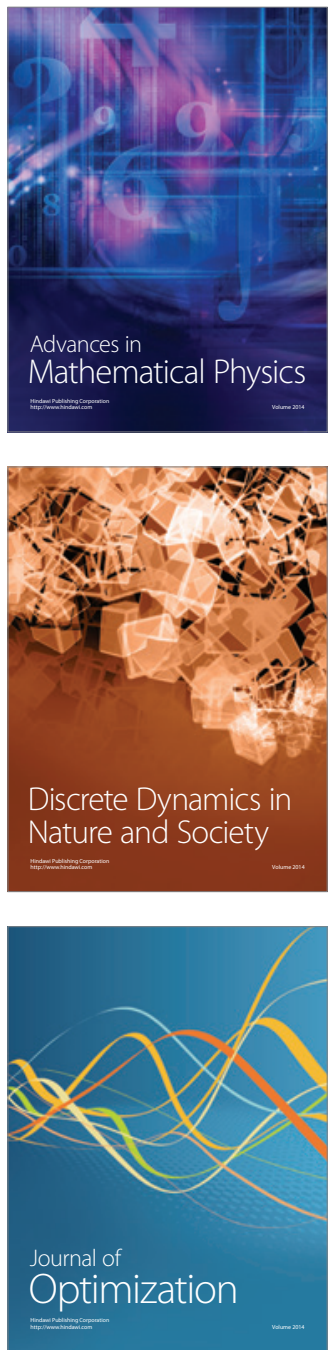